\title{
Out-of-hours care: remuneration alone is no panacea
}

In many developed countries, the delivery of primary care medical services has gradually evolved into two apparently distinct types of service; in-hours and outof-hours $(\mathrm{OOH})$. In-hours care has often been equated to routine GP care during the working week, whereas $\mathrm{OOH}$ care has traditionally been regarded as that provided by GPs for acute problems and emergencies, often by home visiting.

However, the nature of primary medical care provision has also changed in many other ways in the last 20 years. Now primary care providers work in teams and offer a wide range of nursing and medical services, preventive health care, and screening as well as acute care, from increasingly sophisticated clinic premises.

Many primary care service providers, including GPs, offer their routine services for extended hours over and above traditional Monday to Friday clinic times, effectively working shifts to increase patient accessibility to both routine and acute care. Acute care in this setting may well include both unscheduled clinic appointments and home visiting, and it is this type of 'in-hours' same day care that is investigated in the paper by Edwards et al, ${ }^{1}$ examining a successful change in skill mix by expanding the role of the nurse practitioner.

It is useful to consider in-hours care as full-service (routine and acute) primary care, and $\mathrm{OOH}$ care as restricted-service (acute only) primary care, regardless of whether the $\mathrm{OOH}$ care is provided by home visiting, at a clinic, or via a telephone advice service.

In the UK, since the introduction of the 2004 GMS contract, when responsibility for $\mathrm{OOH}$ care passed to primary care trusts, GPs have been able to opt out of providing this type of care for their patients. Many have, and results from Geue et al's paper suggest that those most likely to re-provide $\mathrm{OOH}$ care are most often younger male GPs with dependent families, for whom the desire to raise additional income is attractive. ${ }^{2}$
The prospect of additional income may be one reason to choose to be a reprovider of $\mathrm{OOH}$ care, but there are many factors that make $\mathrm{OOH}$ care different from full service in-hours care. For doctors already working full time, especially if also working into the evening to cover extended hours of opening, the prospect of yet more hours holds little attraction. For those with competing demands from family responsibilities (more likely, but not exclusively, for female GPs) $\mathrm{OOH}$ care is an added burden that can now be alleviated by opting out of re-provision.

More important than the number of hours worked is the differing nature of the work. When dealing with acutely ill patients in usual in-hours clinic circumstances, the support provided by a competent team in familiar surroundings cannot be underestimated. Not only is the patient likely to be known to the practice, but triage, emergency treatment, and further diagnostic assessment can also be arranged most effectively with the backup of a fully staffed clinic.

In the $\mathrm{OOH}$ situation, however, particularly if a home visit or accident scene is involved, the GP must deal with many of these aspects of management alone. The ability to deal with more than one patient at a time is limited, inevitably creating unacceptable waiting times. While there are obvious limitations to $\mathrm{OOH}$ service evaluations that rely heavily on patients' perceptions about waiting times, as reported later in this issue, ${ }^{3}$ they provide an indicator of accessibility and timeliness of treatment. In $\mathrm{OOH}$ situations, clinical decision making is inherently more difficult because patients are much less likely to be known, and options for adequate diagnosis and subsequent patient care are more limited. In rural practice, although patients and their social situation may be more known than in urban areas, travelling distances may be substantial and challenging. In either situation, personal safety may be compromised. On-call work in rural areas may well wear GPs down over time. A recent study investigating male GPs' attitudes to rural general practice in New Zealand suggests that, while the challenge and variety of on-call work may appeal to younger GPs, older GPs, despite being more experienced, describe increasing resentment about the continued need to undertake stressful oncall work. ${ }^{4}$

As Ingram et al describe in their paper in this issue, ${ }^{5}$ GPs vary considerably in their attitude to risk taking, and this affects $\mathrm{OOH}$ referral rates. Variability in risk aversion is also likely to affect GPs' willingness, or otherwise, to tolerate the increased clinical and/or personal risk inherent in $\mathrm{OOH}$ work.

Other factors that influence GPs' hospital referral rates in $\mathrm{OOH}$ situations include time of day and the site where the patient was seen, with patients seen on home visits and between $11 \mathrm{pm}$ and $7 \mathrm{am}$ more likely to be admitted. ${ }^{6}$

In urban areas, the increasing provision of $\mathrm{OOH}$ services from dedicated clinics goes some way to alleviating these difficulties, providing a collegial work environment and extra support when needed. However, the clinical risk is still higher than for in-hours care: almost every consultation is with a new patient, previous patient notes are usually not available, and medication and other errors are more easily made. For risk averse GPs who are given a choice, this is a potentially stressful situation best avoided. $^{7}$

$\mathrm{OOH}$ care remains an essential component of primary medical care, regardless of how it is delivered or who delivers it. Acknowledgement that $\mathrm{OOH}$ care is different from in-hours care is an important first step; however, simply paying more money to those who provide such care is not the whole answer. Structured support in the way of a clinic base, support staff, ability to discuss problems with others, and provision of intermediate levels of care where 
appropriate, are all factors that will not only improve outcomes for patients but also enhance job satisfaction for the health professionals who deliver the care.

Now that responsibility for $\mathrm{OOH}$ care in the UK resides with primary care trusts, there are new and improved opportunities for better provision of safe and effective $\mathrm{OOH}$ care for patients and health professionals alike. $\mathrm{OOH}$ care that mitigates both clinical and personal risks as far as possible is best achieved with a well-structured, well-supported, and adequately resourced approach to acute primary medical care.

\section{Susan Pullon,}

Senior Lecturer, University of Otago Wellington, Wellington, New Zealand.

\section{Lynn McBain,}

Senior Lecturer, University of Otago Wellington, Wellington, New Zealand.

\section{REFERENCES}

1. Edwards M, Bobb C, Robinson SI. Nurse practitioner management of acute in-hours home visit or assessment requests: a pilot study. Br J Gen Pract 2009; 59(558): 7-11.

2. Geue C, Skåtun D, Sutton M. Economic influences on GPs' decisions to provide out-of-hours care. $\mathrm{Br} J \mathrm{Gen}$ Pract 2009; 59(558): 12-17.

3. Campbell J, Roland M, Richards S, et al. User's reports and evaluations of out-of-hours health care and the UK national quality requirements: a crosssectional study. Br J Gen Pract 2009; 59(558): 18-23.

4. Noonan T, Arroll B, Thomas D, et al. When should I do rural general practice? A qualitative study of job/life satisfaction of male rural GPs of differing ages in New Zealand. N Z Med J 2008; 121(1283): 59-67.

5. Ingram JC, Calnan MW, Greenwood RJ, et al. Risk taking in general practice: GP out-of-hours referrals to hospital. Br J Gen Pract 2009; 59(558): 24-28.
6. Rossdale M, Kemple T, Payne S, et al. An observational study of variation in GPs' out-of-hours emergency referrals. Br J Gen Pract 2007; 57(535): 152-154.

7. Calnan M, Payne S, Kemple T, et al. A qualitative study exploring variations in GPs' out-of-hours referrals to hospital. Br J Gen Pract 2007; 57(542): 706-713.

DOI: 10.3399/bjgp09X394770

\section{ADDRESS FOR CORRESPONDENCE}

\section{Susan Pullon}

University of Otago Wellington, Primary Health Care and General Practice, 23a Mein St, Newtown, Wellington, 6021, New Zealand. E-mail: sue.pullon@otago.ac.nz

\section{The contraceptive revolution: some excellent progress but work still to be done}

By 1960 the world's population had grown to around 3 billion people, having taken just 33 years to increase from 2 billion. ${ }^{1}$ Although many agreed that growth rates needed to fall, couples at the time had few reversible contraceptive choices: mainly barrier methods, spermicides, and a few plastic-only and metal-based intrauterine devices (IUDs). Many relied on 'withdrawal'. This was soon to change dramatically because during the 1950s scientists had patented two synthetic progestogens, norethisterone and norethynodrel. ${ }^{2}$ Clinical studies showed that these hormones inhibited ovulation, although some accompanying oestrogen (initially mestranol, now ethinylestradiol) was needed for acceptable breakthrough bleeding and pregnancy rates. The first combined oral contraceptive was marketed in the US in 1960, and in the UK the following year. Many women enthusiastically embraced 'the pill'; for some because it separated contraception from the act of intercourse and for others because it could be used without their partner's knowledge. Early on, however, concerns were expressed about the method's carcinogenic potential, and about reports of associated venous thromboembolic and other cardiovascular events. ${ }^{2}$ Furthermore, the unfolding thalidomide tragedy of the early 1960s provided a powerful reminder of the epidemiological truth that when millions of people use a medicinal product small increases in risk still result in many people affected.

Oral contraception is now one of the most scrutinised medicinal products on the market. Two British investigations that celebrated their 40th anniversaries in 2008 have been major contributors to the evidence base for current clinical practice. Both illustrate the enormous research opportunity of NHS clinical records. The Oxford/Family Planning Association (Oxford/FPA) Study began in 1968, when 17 family planning clinics in England and Scotland started recruiting
17000 white, married women using oral contraception, the IUD or the diaphragm. ${ }^{3}$ The Royal College of General Practitioners' (RCGP) Oral Contraception Study started at the same time, with 1400 GPs throughout the UK recruiting 47000 mainly white, married (or living as married) women, half of whom were using oral contraception. ${ }^{4}$ Both studies have followed up their cohorts through a mixture of clinic or practice reports, personal contact, and the cancer and death notification services of the NHS Central Registries. Each study has provided, in different ways, key insights into the effects of different contraceptives; as well as novel information about other women's health issues. For example, the RCGP study was the first to show that the risk of cardiovascular disease is much higher in pill users who smoke, ${ }^{5}$ especially among older women, and that the risk of hypertension and arterial disease is related to the combined pill's progestogen 